\title{
Retrospective screening of serum and cerebrospinal fluid samples from patients with acute meningo- encephalitis does not reveal past Japanese encephalitis virus infection, Emilia Romagna, Italy, 2011
}

P Gaibani ${ }^{1}$, A C Finarelli ${ }^{2}$, R Cagarelli ${ }^{2}$, A Pierro ${ }^{1}$, G Rossini $^{1}$, M Calzolari ${ }^{3}$, M Dottori $^{3}$, P Bonilauri ${ }^{3}$, M P Landini ${ }^{1}$, VSambri (vittorio.sambri@unibo.it)1

1. Operative Unit of Clinical Microbiology, Regional Reference Centre for Microbiological Emergencies, S. Orsola-Malpighi University Hospital, Bologna, Italy

2. Public Health Authority, Emilia Romagna Region, Bologna, Italy

3. Istituto Zooprofilattico Sperimentale della Lombardia e dell’Emilia-Romagna 'B. Ubertini’ (IZSLER; Experimental veterinary institute of Lombardy and Emilia Romagna), Reggio Emilia, Italy

Gaibani P, Finarelli AC, Cagarelli R, Pierro A, Rossini G, Calzolari M, Dottori M, Bonilauri P, Landini MP, Sambri V. Retrospective screening of serum and

cerebrospinal fluid samples from patients with acute meningo-encephalitis does not reveal past Japanese encephalitis virus infection, Emilia Romagna, Italy, 2011. Euro Surveill. 2012;17(35):pii=20257. Available online: http://www.eurosurveillance.org/ViewArticle.aspx?Articleld=20257

To the editor:

A recent publication by Ravanini et al reported the detection of a Japanese encephalitis virus (JEV) RNA sequence in one pool of Culex pipiens mosquitoes, collected in 2010 in the province of Bologna (EmiliaRomagna region) [1]. In that study, a partial genomic sequence of $167 \mathrm{bp}$ was shown to have $100 \%$ similarity to the $\mathrm{NS}_{5}$ region of the JEV genotype III genome. Confirmatory specific RT-PCR targeting the envelope, $\mathrm{NS}_{3}$ and NS 5 regions of the JEV genome and attempts at virus isolation in cell culture were repeatedly negative.

Another retrospective study by Platonov et al., conducted in bird samples collected 10 years earlier, had investigated the presence of JEV RNA [2]. In particular, the authors had amplified a 215 bp fragment of the flavivirus $\mathrm{NS}_{5}$ gene in six of seven birds positive for JE group antigens in immunohistochemistry. Based on these findings, the possible introduction of JEV in a new area, and in particular in southern Europe, has been hypothesised and discussed [1-3]. We think that the detection of genomic RNA fragments (167 and 157 bp from the flavivirus NS5 gene and 552 bp from the JEV $E$ gene in the above papers) cannot confirm the presence and circulation of JEV in Europe, and Italy in particular.

We therefore investigated the possibility of human cases of neurological infection caused by JEV in a retrospective serological study. Sera and cerebrospinal fluid (CSF) samples were obtained from 38 subjects with clinical symptoms of acute meningoencephalitis, collected in the province of Bologna between 1 January and 31 December 2011. The specimens were referred to the Regional Reference Centre for Microbiological Emergencies (Centro di Riferimento Regionale per le Emergenze Microbiologiche; CRREM) at St. Orsola-Malpighi Hospital, Bologna, as part of the regional surveillance programme for West Nile virus infections that was started in 2009 [4]. To evaluate the presence of JEV-specific IgM or IgG antibodies, the samples were tested by a commercial indirect immunofluorescence assays (IFA, Euroimmun, Lübeck, Germany). Following the guidelines issued by the World Health Organization, the diagnosis of JEV should preferably be achieved by detection of specific IgM antibodies in the CSF, while the detection of the JEV genome (in serum, plasma, blood or (SF), or of JEV-related antigens in tissue by immunofluorescence/immunohistochemistry or virus isolation can be used in addition or as confirmatory test [5]. None of these CSF or serum samples was IgG- or IgM-positive by IFA, indicating that none of them contained specific antibodies against JEV.

In conclusion, our findings clearly indicate that no human cases of meningoencaphilitis due to JEV occurred in Bologna in the months following the reported detection of a short JEV genomic sequence in C. pipiens pool collected in the same area [1]. A recent study conducted on different mosquito species collected between 2007 and 2010 in a larger area of the Emilia Romagna region, identified the presence of several flaviviruseses closely related to JEV by RT-PCR targeting the flavivirus $\mathrm{NS}_{5}$ region; the analysis of these sequences, however, was unable to identify precisely and without doubt whether or not they corresponded to the JEV genome [6]. Moreover, 269,686 mosquitoes (of which 233,074 were C. pipiens) and 1,486 wild birds (418 of which were collected passively) obtained in 2011 in the context of the regional surveillance plan in Emilia Romagna, were tested with the described flavivirus-specific RT-PCR, and no JEV sequences were detected (data not shown). In addition, we are 
at present still monitoring flaviviruses in mosquitoes and birds, and further amplicons belonging to the JEV genome have not been detected.

Based on these findings, the hypothesis proposed by Platonov et al. [2] of a new flavivirus, closely related to JEV, appears the most consistent. However, our findings cannot definitively exclude the possible circulation of JEV or other human pathogenic JEV-related flaviviruses in the province of Bologna and consequently, extensive human, entomological and veterinary screening with molecular techniques, will be carried out, to confirm or to rule out the possible circulation of new flaviviruses in Italy.

\section{References}

1. Ravanini P, Huhtamo E, Ilaria V, Crobu M, Nicosia A, Servino L, et al. Japanese encephalitis virus RNA detected in Culex pipiens mosquitoes in Italy. Euro Surveill.

2012;17(28): pii=20221. Available from: http://www.

eurosurveillance.org/ViewArticle.aspx?Articleld=20221

2. Platonov AE, Rossi G, Karan LS, Mironov KO, Busani L, Rezza G. Does the Japanese encephalitis virus (JEV) represent a threat for human health in Europe? Detection of JEV RNA sequences in birds collected in Italy. Euro Surveill. 2012; 17(32): pii=20241. Available from: http://www.eurosurveillance.org/ViewArticle. aspx?Articleld $=20241$

3. Zeller $\mathrm{H}$. Is Japanese encephalitis emerging in Europe? Euro Surveill. 2012;17(32): pii=20242. Available from: http://www. eurosurveillance.org/ViewArticle.aspx?Articleld=20242

4. Angelini P, Tamba M, Finarelli AC, Bellini R, Albieri A, Bonilauri $P$, et al. West Nile virus circulation in Emilia-Romagna, Italy: the integrated surveillance system 2009. Euro Surveill. 2010; 15(16): pii=19547. Available from: http://www.eurosurveillance. org/ViewArticle.aspx?Articleld=19547

5. World Health Organization (WHO). Manual for the laboratory diagnosis of Japanese encephalitis virus infection. Geneva: WHO; March 2007. Available from: http://www.who.int/ immunization_monitoring/Manual_lab_diagnosis_JE.pdf

6. Calzolari M, Zé-Zé L, Růžek D, Vázquez A, Jeffries C, Defilippo $\mathrm{F}$, et al. Detection of mosquito-only flaviviruses in Europe. J Gen Virol. 2012;93(Pt 6):1215-25. 